\title{
Budget Impact of 12-Month Fixed Treatment Duration Venetoclax in Combination with Obinutuzumab in Previously Untreated Chronic Lymphocytic Leukemia Patients in the United States
}

\author{
Sang Kyu Cho ${ }^{1,2} \cdot$ Beenish S. Manzoor ${ }^{2}(1) \cdot$ Kavita R. Sail $^{2} \cdot$ Hélène Parisé $^{3} \cdot$ Arliene Ravelo $^{4} \cdot$ Sheila Shapouri $^{4}$. \\ Tatyana Kapustyan $^{2} \cdot$ Simon Sharmokh ${ }^{2} \cdot$ Suchin Virabhak ${ }^{3} \cdot$ Matthew S. Davids $^{5} \cdot$ Scott Johnson $^{3}$
}

Published online: 8 May 2020

(c) The Author(s) 2020

\begin{abstract}
Objectives This study aimed to assess the total cost of care (TCC) and budget impact of introducing 12-month fixed duration venetoclax + obinutuzumab $(\mathrm{VEN}+\mathrm{G})$ as first-line treatment for chronic lymphocytic leukemia (CLL) from the perspective of a US health plan with 1,000,000 (1M) members.

Methods The 3-year model included the following comparators: fludarabine + cyclophosphamide + rituximab (FCR), bendamustine + rituximab (BR), obinutuzumab + chlorambucil (GClb), ibrutinib (Ibr), and Ibr+Rituximab/obinutuzumab [Ibr+R/ Ibr+G]). TCC included US-specific costs associated with treatment (i.e., drug, administration, and wastage), adverse events, routine care, and monitoring. Dosing and safety data were drawn from clinical trials and US package inserts. Budget impact outcomes were presented on an absolute and per-member per-month (PMPM) basis. Sensitivity analyses explored uncertainty in influential parameters, including scenarios testing the duration of treat-to-progression agents.

Results Over the 3-year time horizon, introducing VEN+G in a $1 \mathrm{M}$-member health plan resulted in total cost savings of $\$ 1,550,663$ (PMPM - \$0.04), compared to a scenario without VEN+G. The fixed 12-month duration of VEN+G contributed to this cost saving by reducing cumulative treatment costs compared with Ibr-based regimens. By year 3 , the cumulative difference in TCC of VEN+G compared with Ibr, Ibr+G, and Ibr+R amounted to - \$300,942, $-\$ 367,001$, and - \$369,784, respectively. Extensive sensitivity analyses supported the base case findings.

Conclusions Introducing VEN+G among first-line CLL treatments to a US health plan resulted in cost savings compared to a plan with chemoimmunotherapies and Ibr-based therapies only. Economic benefits of VEN+G, a novel agent with fixed treatment duration, coupled with proven clinical benefits should help inform formulary adoption decisions and treatment recommendations.
\end{abstract}

Electronic supplementary material The online version of this article (https://doi.org/10.1007/s40273-020-00919-1) contains supplementary material, which is available to authorized users.

Beenish S. Manzoor

beenish.manzoor@abbvie.com

1 University of Southern California School of Pharmacy, Los Angeles, USA

2 AbbVie Inc., North Chicago, IL, USA

3 Medicus Economics, LLC, Milton, MA, USA

4 Genentech, South San Francisco, CA, USA

5 Department of Medical Oncology, Dana-Farber Cancer Institute, Boston, MA, USA

\section{Key Points for Decision Makers}

Assuming an uptake of $21.4 \%$ by year 3 , introducing venetoclax + obinutuzumab $(\mathrm{VEN}+\mathrm{G})$ as first-line treatment for chronic lymphocytic leukemia (CLL) is associated with a total cost saving of $\$ 1,550,663$ over a 3-year period, compared to a scenario without VEN+G. This translated to a per-member per-month saving of $\$ 0.04$.

In addition, there was a reduction of $49-54 \%$ in the 3 -year cumulative total cost of care for $\mathrm{VEN}+\mathrm{G}$ compared to ibrutinib-based regimens (including monotherapy).

These cost savings are due to the fixed 12-month treatment of VEN+G, compared to ibrutinib-based regimens, which are given continuously until disease progression or unacceptable toxicity. 


\section{Introduction}

Chronic lymphocytic leukemia (CLL) is one of the two most common forms of leukemia in adults [1], with an estimated incidence of 20,720 new cases in 2019 and approximately 178,206 patients living with the disease in the United States (US) [2]. CLL is a slow, progressive disease, mainly in the blood, bone marrow, and lymph nodes [3, 4]. CLL is more prevalent in older adults, with a median age of diagnosis at 72 years [3], who frequently present with comorbidities that are associated with a poorer prognosis $[5,6]$. Due to its slow progression and the poorer prognosis of older patients, CLL poses an important economic burden to patients, payers, and society, in addition to negatively impacting the quality of life of patients [7-9]. Chen et al. [9] estimated that with the introduction of oral targeted therapies in second- and firstline settings, the annual cost (in 2015 US dollars [USD]) of CLL management in the US would increase from $\$ 0.74$ billion in 2011 to $\$ 5.13$ billion (593\%) in 2025 .

Current pharmacotherapies for first-line CLL include chemoimmunotherapy (i.e., fludarabine, cyclophosphamide plus rituximab [FCR], bendamustine plus rituximab [BR], and obinutuzumab plus chlorambucil [GClb]) and novel targeted therapies, such as ibrutinib (Ibr) and Ibr with rituximab (Ibr+R) or with obinutuzumab (Ibr+G) [10]. Chemoimmunotherapy regimens have a 24-month progression-free survival (PFS) between 60 and 74\% [5, 11], fixed treatment durations of up to six cycles [5, 6], with drug costs ranging from $\$ 57,000$ to $\$ 101,000$ for a commercially insured individual (Truven Health Analytics, Ann Arbor, MI, USA). However, the limited drug exposure due to toxicities translates to poor efficacy $[5,11,12]$. In contrast, novel targeted Ibr-based regimens (Ibr, Ibr+R, and Ibr+G) have a 24-month PFS between 73 and $89 \%[11,13]$ and are dosed continuously until unacceptable toxicity or disease progression [14], often for several years, and the annual drug cost of Ibr monotherapy is approximately $\$ 169,000$ for a commercially insured individual (Truven Health Analytics, Ann Arbor, MI, USA). Although Ibr-based regimens have better efficacy than chemoimmunotherapies [11, 13, 15], novel targeted therapies that are administered to progression could strain the budget of both private and government payers, and their copayments could add a substantial burden to patients [8, 9]. Reducing the economic burden of first-line CLL treatment remains an unmet need [9].

In May 2019, the US Food and Drug Administration (FDA) approved venetoclax (Venclexta®), an oral B-cell lymphoma-2 inhibitor, in combination with obinutuzumab (Gazyva®) for a 12-month, fixed treatment duration in previously untreated patients with CLL [16]. The approval of venetoclax in combination with obinutuzumab $(\mathrm{VEN}+\mathrm{G})$ was based on data from a phase 3 randomized controlled trial (RCT), CLL14 (NCT02242942), that evaluated the efficacy and safety of $\mathrm{VEN}+\mathrm{G}$ compared to $\mathrm{GClb}$ in patients with previously untreated CLL with coexisting medical conditions [12]. Over a median follow-up of 28 months, VEN+G reduced the risk of progression or death by $67 \%$ compared to GClb (hazard ratio [HR] 0.33, 95\% confidence interval [CI] $0.22-0.51 ; p<0.0001$ ) [16]. The Kaplan-Meier estimate of the percentage of patients with PFS at 24 months was significantly higher in the $\mathrm{VEN}+\mathrm{G}$ group than in the GClb group: $88.2 \%$ (95\% CI 83.7-92.6) as compared to $64.1 \%$ (95\% CI 57.4-70.8) [12].

The CLL14 trial supports the clinical value of VEN+G, but further evidence on its economic benefit is needed to inform formulary adoption and treatment recommendations. The fixed duration of $\mathrm{VEN}+\mathrm{G}$ as compared to existing targeted therapies could alleviate the financial burden of CLL on payers and patients. Therefore, we developed a budget impact model (BIM) to analyze the total cost of care (TCC) and budget impact of introducing $\mathrm{VEN}+\mathrm{G}$ as first-line treatment for CLL from a US healthcare payer perspective.

\section{Methods}

\subsection{Model Structure}

A BIM was developed to estimate two different potential scenarios over a 3-year time horizon: one where the current treatment landscape continued without the introduction of $\mathrm{VEN}+\mathrm{G}$ (current scenario), and another where VEN+G was an available option in the treatment landscape (projected scenario). A comparison of the current and projected scenarios provided an estimate of the budget impact of $\mathrm{VEN}+\mathrm{G}$ being covered and reimbursed over a given time horizon. The model was developed in 28-day cycles (4-week cycles). The analyses were conducted over a 3-year time horizon (5-year in a scenario analysis), where each year had a duration of 364 days (i.e., 13 cycles of 28 days).

The model calculated the TCC for each regimen and included treatment costs (drug, administration, and wastage), adversel event (AE) costs, routine costs of care (CLL related and all cause), and tumor lysis syndrome (TLS) monitoring costs. The budget impact for each of the first 3 years and the overall 3-year budget impact were assessed. Budget outcomes were presented in absolute and net terms and included per-member per-month/year (PMPM/PMPY) calculations. All costs are presented in 2019 USD, and drug acquisition costs are current as of January 2020. Where applicable, the medical care component of the consumer price index (CPI) was used to inflate costs to 2019 USD [17].

The model was developed in Microsoft Excel 2016 (Microsoft Corporation, Redmond, WA, USA) and complied with the recommendations of the International Society for 
Pharmacoeconomics and Outcomes Research Principles of Good Practice for Budget Impact Analysis [18].

\subsection{Target Population}

The target population in the model included previously untreated adult patients with CLL and was based on the CLL incidence rate estimated from the Surveillance, Epidemiology, and End Results Program (SEER) [19]. More specifically, incidence of CLL among patients 18-64 years old was obtained from SEER and weighted by the age distribution from the 2017 National Population Projections Datasets of the US Census Bureau (year selected: 2020) [20], whereas the incidence of CLL among patients 65 years and older was directly obtained from SEER. Furthermore, to derive the number of patients to be potentially treated with VEN+G, we assumed patients 18-64 years old versus 65 years and older were covered by 'Commercial' (Blue Cross, commercial carriers, private health maintenance organizations [HMOs], and preferred provider organizations [PPOs], as defined in https://hcupnet.ahrq.gov) and Medicare, respectively. We assumed at the start of the model that there were $1,000,000$ health plan patients, of whom $20 \%$ were covered by Medicare (Supplementary Table 1; see the electronic supplementary material). As this distribution affected both the number of patients entering the model and the associated costs, we tested it in scenario analyses. The derivation of the target population is depicted in Fig. 1.

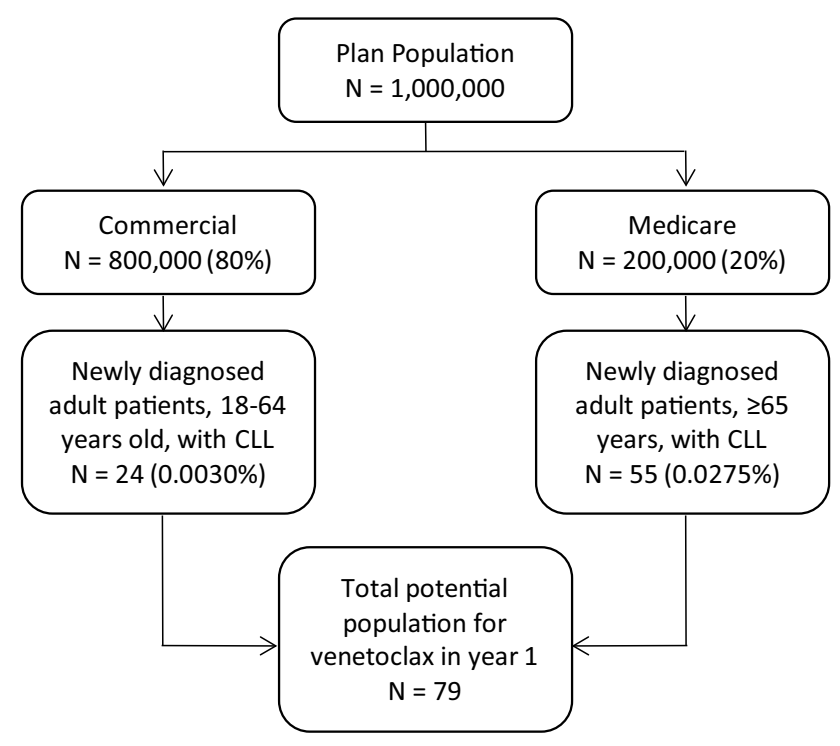

Fig. 1 Derivation of the target population in year 1. All $N \mathrm{~s}$ are rounded to the nearest whole numbers; thus totals may not add up, due to rounding error. $C L L$ chronic lymphocytic leukemia, $N$ number

\subsection{Comparators and Market Share}

Comparators were chosen based on current market share and the anticipated treatment landscape in the first-line CLL indication. The dosing of each comparator was based on US package insert (PI) or trial publication, as follows: GClb [13, 21], Ibr [14], Ibr+R [11], Ibr+G [14], FCR [5], and BR [5]. In terms of treatment duration applied in the model, $\mathrm{GClb}$, FCR, and BR had a fixed duration of six cycles. VEN+G had a 12-month fixed duration (obinutuzumab is administered for six cycles) [16], whereas Ibr is a treat-to-progression agent. Since PFS and overall survival (OS) were not considered in the model base case, Ibr treatment was administered continuously. Scenario analyses limiting its duration were conducted. Alternatively, a scenario applying PFS and OS to all regimens was included.

Patients were distributed to different treatment regimens according to specified market shares that were based on projections made by the sponsors of VEN $+\mathrm{G}$, namely AbbVie Inc. and Genentech (Supplementary Table 2; see the electronic supplementary material).

\subsection{Cost Components}

\subsubsection{Total Treatment Costs}

Total treatment costs included drug costs, wastage costs, and administration costs; these were applied for as long as treatment was given.

Drug costs were based on wholesale acquisition cost (WAC) prices (Truven Health Analytics, Ann Arbor, MI, USA) (Supplementary Table 3; see the electronic supplementary material). Wastage was assumed only for intravenous (IV) drugs, where a discrepancy between vial size and the actual dosage required resulted in wastage. Oral medications incurred no wastage. In the case where multidose vials were available (i.e., bendamustine), we assumed there was no wastage.

Only IV treatments were assumed to incur administration costs. These were calculated based on the time needed to administer the drug, taking into account information on dosing and infusion rate found in US PIs [22-24]. We applied a conservative approach where the standard infusion rate (i.e., ignoring infusion-related complications) was used. The time required for administering each drug compound was then matched to its relevant Current Procedural Terminology (CPT) code. Finally, unit cost for each CPT code was based on a routine cost-of-care analysis using Truven MarketScan databases from 01/01/2000 to 06/30/2017 (data on file, AbbVie Inc., 2018) (Supplementary Table 4$)$. The resulting administration costs are depicted in Supplementary Table 5. 
Table 1 Total treatment cost per cycle. Sources: Truven Health Analytics, Ann Arbor, MI, USA, accessed January 28, 2020; Venclexta ${ }^{\circledR}$ US PI [16]; Gazyva ${ }^{\circledR}$ US PI [22]; Bendeka ${ }^{\circledR}$ US PI [23]; Rituxan ${ }^{\circledR}$ US PI [24]; https://emedicine.medscape.com/article/2005390-overv iew, accessed February 11, 2020

\begin{tabular}{|c|c|c|c|c|}
\hline \multirow[t]{2}{*}{ Regimen } & \multirow[t]{2}{*}{ Cycle } & \multicolumn{3}{|c|}{ Treatment cost } \\
\hline & & Drug & Wastage & Administration $^{\mathrm{a}}$ \\
\hline \multirow[t]{5}{*}{ VEN+G } & Cycle 1 & $\$ 20,091$ & $\$ 0$ & $\$ 3141$ \\
\hline & Cycle 2 & $\$ 12,028$ & $\$ 0$ & $\$ 785$ \\
\hline & Cycles 3-6 & $\$ 18,123$ & $\$ 0$ & $\$ 785$ \\
\hline & Cycles 7-12 & $\$ 11,474$ & $\$ 0$ & $\$ 0$ \\
\hline & Cycle 13+ & $\$ 0$ & $\$ 0$ & $\$ 0$ \\
\hline \multirow[t]{3}{*}{$\mathrm{GClb}$} & Cycle 1 & $\$ 20,860$ & $\$ 12$ & $\$ 3141$ \\
\hline & Cycles 2-6 & $\$ 7562$ & $\$ 12$ & $\$ 785$ \\
\hline & Cycle 7+ & $\$ 0$ & $\$ 0$ & $\$ 0$ \\
\hline Ibr & Cycle 1+ & $\$ 12,966$ & $\$ 0$ & $\$ 0$ \\
\hline \multirow[t]{4}{*}{$\mathrm{Ibr}+\mathrm{R}$} & Cycle 1 & $\$ 12,966$ & $\$ 0$ & $\$ 0$ \\
\hline & Cycle 2 & $\$ 39,461$ & $\$ 3570$ & $\$ 2649$ \\
\hline & Cycles 3-6 & $\$ 19,590$ & $\$ 893$ & $\$ 621$ \\
\hline & Cycle 7+ & $\$ 12,966$ & $\$ 0$ & $\$ 0$ \\
\hline \multirow[t]{3}{*}{$\mathrm{Ibr}+\mathrm{G}$} & Cycle 1 & $\$ 32,914$ & $\$ 0$ & $\$ 3141$ \\
\hline & Cycles 2-6 & $\$ 19,615$ & $\$ 0$ & $\$ 785$ \\
\hline & Cycle 7+ & $\$ 12,966$ & $\$ 0$ & $\$ 0$ \\
\hline \multirow[t]{3}{*}{ FCR } & Cycle 1 & $\$ 7694$ & $\$ 961$ & $\$ 2058$ \\
\hline & Cycles 2-6 & $\$ 9902$ & $\$ 632$ & $\$ 2058$ \\
\hline & Cycle 7+ & $\$ 0$ & $\$ 0$ & $\$ 0$ \\
\hline \multirow[t]{3}{*}{$\mathrm{BR}$} & Cycle 1 & $\$ 14,995$ & $\$ 893$ & $\$ 1105$ \\
\hline & Cycles 2-6 & $\$ 17,203$ & $\$ 564$ & $\$ 1105$ \\
\hline & Cycle 7+ & $\$ 0$ & $\$ 0$ & $\$ 0$ \\
\hline
\end{tabular}

$B R$ bendamustine + rituximab, $C P T$ Current Procedural Terminology, $F C R$ fludarabine + cyclophosphamide + rituximab, $G$ Gazyva ${ }^{\circledR}$ (obinutuzumab), $G C l b$ obinutuzumab + chlorambucil, $I b r$ ibrutinib, $R$ rituximab, $V E N$ venetoclax

${ }^{a}$ Unit cost for relevant CPT codes from a routine cost-of-care analysis using Truven MarketScan databases from 01/01/2000 to 06/30/2017 (data on file, 2018)

Table 1 tabulates total treatment costs (i.e., drug, wastage, and administrative costs) for each therapy by treatment cycle.

\subsubsection{Adverse Events}

AEs of grade $\geq 3$ severity and occurring in at least $5 \%$ of patients treated with any regimen were included, except those listed under a broader category (e.g., vascular disorder). Specifically, grade 3 AEs are "severe or medically significant but not immediately life-threatening; hospitalization or prolongation of hospitalization indicated; disabling; limiting self-care ADL" (where ADL stands for activities of daily living); grade 4 AEs are "life-threatening consequences; urgent intervention indicated"; grade 5 AEs are "death related to AE" [25]. The AE rates, presented in Table 2, were extracted from relevant publications or US PIs and were applied once in the first cycle of the model. Of note, AE rates not reported for a given regimen were assumed to be zero.

The costs of AEs were derived from the Healthcare Cost and Utilization Project (HCUP) (Hospital Inpatient National Sample 2015 available at https://hcupnet.ahrq.gov/, accessed February 10, 2020) based on the appropriate International Classification of Diseases, Ninth Revision (ICD-9) codes of each AE. Separate Medicare and Commercial costs were obtained (Supplementary Table 6; see the electronic supplementary material). The model then computed a weighted average of the Medicare and Commercial costs based on the proportion of Medicare patients assumed (e.g., 20\% in the base case).

\subsubsection{Routine Costs of Care}

Routine costs of care comprised CLL management costs (i.e., routine office/outpatient visits and blood tests) and total all-cause healthcare costs, exclusive of drug therapy costs. The CLL management costs were based on Chen et al. (2017) [9] and consist of physician visit and blood tests after a commonly practiced follow-up schedule. More specifically, for chemoimmunotherapy, this schedule was weekly for cycle 1 , every 2 weeks for cycles $2-6$, and then every 1 , 3 , and 6 months until years 1, 3, and afterward, respectively. For oral targeted therapies, it was weekly for 2 months, every month until month 6, and every 3 months afterward (Supplementary Table 7; see the electronic supplementary material). The resulting total CLL management costs in year 1 were $\$ 3561$ for chemoimmunotherapies and $\$ 2374$ for oral targeted therapies. The CLL management costs fell to $\$ 678$ from year 2 to 5 for oral targeted therapies. For chemoimmunotherapies, costs in years 2-3 and 4-5 were $\$ 678$ and $\$ 339$, respectively. For each regimen, total all-cause healthcare costs, excluding drug costs, amounting to $\$ 2684$ per cycle were also included, based on Wang et al. (2018) [26].

Routine costs of care were applied continuously throughout the model, as mortality was excluded in the base case.

\subsubsection{TLS Monitoring Costs}

Per the Venclexta ${ }^{\circledR}$ US PI [16], TLS monitoring is required, including prophylaxis medication, and hydration; as overall risk increases, more intensive measures, such as IV hydration, and hospitalization are recommended. Thus, in the base case, TLS monitoring costs, aligned with the algorithm described in the US PI [16], were applied for VEN+G. Regarding the risk for TLS, $13.4 \%, 64.4 \%$, and $22.2 \%$ were at low, medium, and high risk, respectively, based on the 
Table 2 Adverse event rates and associated costs. Sources: Fischer et al. [12]; Barr et al. [15]; Woyach et al. [11]; Imbruvica ${ }^{\circledR}$ US PI [14]; Eichhorst et al. [5]

\begin{tabular}{|c|c|c|c|c|c|c|c|}
\hline Adverse event & $\mathrm{VEN}+\mathrm{G}$ & $\mathrm{GClb}$ & $\mathrm{Ibr}^{\mathrm{b}}$ & $\mathrm{Ibr}+\mathrm{R}^{\mathrm{c}}$ & $\mathrm{Ibr}+\mathrm{G}$ & FCR & BR \\
\hline \multicolumn{8}{|l|}{ Adverse event rate ${ }^{\mathrm{a}}(\%)$} \\
\hline Anemia & 8.0 & 6.5 & 6.7 & 5.5 & 4.0 & 13.6 & 10.4 \\
\hline Febrile neutropenia & 5.2 & 3.7 & 0.0 & 0.5 & 0.0 & 0.0 & 0.0 \\
\hline Infusion related reaction & 9.0 & 10.3 & 0.0 & 0.0 & 2.0 & 0.0 & 0.0 \\
\hline Leukopenia/leukocytopenia & 2.4 & 4.7 & 0.0 & 0.0 & 0.0 & 80.6 & 48.6 \\
\hline Neutropenia & 52.8 & 48.1 & 11.9 & 21.4 & 39.0 & 84.2 & 59.0 \\
\hline Pneumonia & 4.2 & 3.7 & 6.0 & 0.0 & 9.0 & 12.2 & 8.6 \\
\hline Thrombocytopenia & 13.7 & 15.0 & 2.2 & 4.9 & 19.0 & 21.5 & 14.4 \\
\hline Allergic conditions & 0.0 & 0.0 & 0.0 & 0.0 & 0.0 & 5.4 & 9.7 \\
\hline Atrial fibrillation & 0.0 & 0.0 & 4.4 & 4.9 & 5.0 & 0.0 & 0.0 \\
\hline Hypertension & 0.0 & 0.0 & 5.2 & 33.5 & 4.0 & 0.0 & 0.0 \\
\hline Sepsis & 0.0 & 0.0 & 0.0 & 3.3 & 0.0 & 3.2 & 1.8 \\
\hline Viral infection & 0.0 & 0.0 & 0.0 & 0.0 & 0.0 & 8.6 & 3.6 \\
\hline Total adverse event cost ${ }^{\mathrm{d}}$ & $\$ 11,097$ & $\$ 10,529$ & $\$ 3734$ & $\$ 7313$ & $\$ 9532$ & $\$ 24,118$ & $\$ 16,294$ \\
\hline
\end{tabular}

$B R$ bendamustine + rituximab, $F C R$ fludarabine + cyclophosphamide + rituximab, $G$ Gazyva ${ }^{\circledR}$ (obinutuzumab), $G C l b$ obinutuzumab + chlorambucil, $I b r$ Ibrutinib, $R$ rituximab, $V E N$ venetoclax

${ }^{a}$ Adverse events not reported were assumed to be zero

${ }^{b}$ First year rate of thrombocytopenia taken from text of RESONATE-2 trial publication (Barr et al. [15]) and is likely to be underestimated, as the corresponding rates for anemia and neutropenia reported in the text were lower than those reported in Table S2

${ }^{c}$ Data for grade 3 and 4 decreases in platelet count (neutrophil count) used in the absence of grade $\geq 3$ thrombocytopenia (neutropenia) rates, respectively. Data for allergic conditions drawn from 'allergic reaction' in Table S8 of the ALLIANCE trial publication (Woyach et al. [11])

${ }^{\mathrm{d}}$ Calculated as the product of the adverse event rate and its respective cost, where cost is a weighted average between the cost for Commercial and Medicare. Adverse event costs are applied once in year 1
CLL14 trial data [12]. It is important to note that TLS tumor burden assessment was done prior to initiation of any therapy in the CLL14 trial, whereas at the time of the first venetoclax dose, patients received three doses of obinutuzumab, which may have impacted their risk of TLS, and hence TLS prophylaxis. The TLS costs stratified by tumor burden and payer type are detailed in Supplementary Table 8 (see the electronic supplementary material). The total TLS costs, applied once in the first year of the BIM, was $\$ 15,686$ for Commercial patients and $\$ 17,362$ for Medicare patients. We conservatively assumed that only VEN+G incurred TLS monitoring costs. This is tested in scenario analyses.

\subsection{Sensitivity Analyses}

Sensitivity analyses were conducted around the budget impact, including deterministic sensitivity analysis (DSA) and scenario analyses. Parameters varied in the DSA are detailed in Supplementary Table 9 (see the electronic supplementary material). Additional scenario analyses were conducted to test structural assumptions. First, the time horizon was extended to 5 years. Second, the treatment duration of Ibr-based therapies was restricted to 18 months, 24 months, or 30 months, compared to the base case, where Ibr was assumed to be given continuously. Third, the base case assumes neither mortality nor progression occur in the model. To relax this assumption, PFS/OS data were used to model patient progression and mortality [27]. Fourth, the base case assumed that only high tumor burden patients treated with VEN+G were hospitalized for TLS prophylaxis measures. We tested this assumption in scenario analysis by assuming that $100 \%$ of medium TLS risk patients were also hospitalized. Fifth, the payer mix was varied by assuming Medicare patients formed the majority of patients $(80 \%)$, compared to the base case, where they comprised $20 \%$ of patients. Additionally, we tested two scenarios: Commercial patients only and Medicare patients only. Finally, we explored a scenario where instead of WAC prices, Big4 prices from the Veterans Affairs (VA) national acquisition catalog were used [28], as these are conservative relative to prices in Commercial plans. We found that on average, Big4 prices are $64-72 \%$ of WAC prices.

\section{Results}

\subsection{Budget Impact Results}

Table 3 presents the overall current and projected budget as well as the budget impact (total, PMPM, and PMPY) 
associated with the addition of VEN+G for first-line treatment in previously untreated CLL patients. The budget impact for each of the first 3 years and the overall 3-year budget impact are shown in Table 3. The introduction of $\mathrm{VEN}+\mathrm{G}$ to the first-line treatment landscape resulted in cost savings of $\$ 1,550,663$ to a 1 -million-member health plan over a 3-year time horizon. This translated to an average PMPM (PMPY) budget impact of $-\$ 0.04(-\$ 0.52)$ over the same period. In fact, adding VEN+G to the formulary of a health plan led to cost savings as early as year 2 (Table 3 ).

\subsection{Annual and Cumulative TCC}

The annual TCC per patient by regimen for the first 3 years is shown in Fig. 2 (5-year: Supplementary Fig. 1; see the electronic supplementary material). Treatment with chemoimmunotherapy regimens (namely GClb, FCR, and BR) costs less than treatment with targeted therapies (i.e., $\mathrm{VEN}+\mathrm{G}$ and Ibr-based regimens). While the annual TCC per patient for treatment with VEN $+\mathrm{G}$ in year 1 was comparable to other targeted therapies, from year 2 onwards, the TCC of VEN+G fell to $\$ 35,570$, which is entirely attributed to the routine cost of care (Fig. 3). On the other hand, the TCC per patient of Ibr-based regimens remained high $(\$ 204,130$ / annum from year 2) due to the cost of continued treatment until disease progression.
Cumulatively over the 3-year time horizon, the TCCs per patient were lowest for GClb $(\$ 185,931)$, FCR $(\$ 207,387)$, and BR $(\$ 237,238)$, followed by VEN+G $(\$ 316,877)$, Ibr $(\$ 617,819)$, Ibr+G $(\$ 683,878)$, and Ibr+R (\$686,660) (Fig. 3). Over a 3-year time horizon, there was a $49-54 \%$ reduction in cumulative TCC with VEN $+\mathrm{G}$ compared to Ibr-based regiments due to the fixed 12-month duration of VEN+G. By year 3, when compared to $\mathrm{VEN}+\mathrm{G}$, the cumulative difference in TCC per patient amounted to $-\$ 300,942$ versus $\mathrm{Ibr},-\$ 367,001$ versus $\mathrm{Ibr}+\mathrm{G}$, and $-\$ 369,784$ versus Ibr+R (Fig. 3).

Figure 3 also shows cumulative TCC broken down into its cost components: treatment costs accounted for the largest share of cumulative TCC for Ibr-based regimens $(\sim 80 \%)$. On the other hand, routine cost of care formed the largest cost component for chemoimmunotherapies (between 46 and 59\%). For VEN+G, treatment costs made up about $57 \%$ of the 3 -year cumulative TCC, while routine cost of care accounted for $34 \%$.

\subsection{Sensitivity Analyses}

The scenario analysis results were aligned with the base case findings (Table 4). The model time horizon and payer channel had the most impact on the incremental budget associated with the inclusion of VEN+G over 3 years.
Table 3 Total budget in the current vs. projected scenario and budget impact
Fig. 2 Comparison of annual total cost of care per patient by choice of therapy. $B R$ bendamustine + rituximab, $F C R$ fludarabine + cyclophosphamide + rituximab, $G$ Gazyva ${ }^{\circledR}$ (obinutuzumab), GClb obinutuzumab + chlorambucil, Ibr ibrutinib, $R$ rituximab, VEN venetoclax

\begin{tabular}{lllll}
\hline Budget & Year 1 & Year 2 & Year 3 & Total/average \\
\hline Current scenario & $\$ 15,939,000$ & $\$ 28,258,531$ & $\$ 41,066,047$ & $\$ 85,263,579$ \\
Projected scenario & $\$ 16,041,767$ & $\$ 28,144,468$ & $\$ 39,526,681$ & $\$ 83,712,916$ \\
Budget impact & $\$ 102,767$ & $-\$ 114,063$ & $-\$ 1,539,367$ & $-\$ 1,550,663$ \\
PMPM budget impact & $\$ 0.0086$ & $-\$ 0.0095$ & $-\$ 0.1283$ & $-\$ 0.0431$ \\
PMPY budget impact & $\$ 0.1028$ & $-\$ 0.1141$ & $-\$ 1.5394$ & $-\$ 0.5169$ \\
\hline
\end{tabular}

$P M P M$ per-member per-month, $P M P Y$ per-member per-year

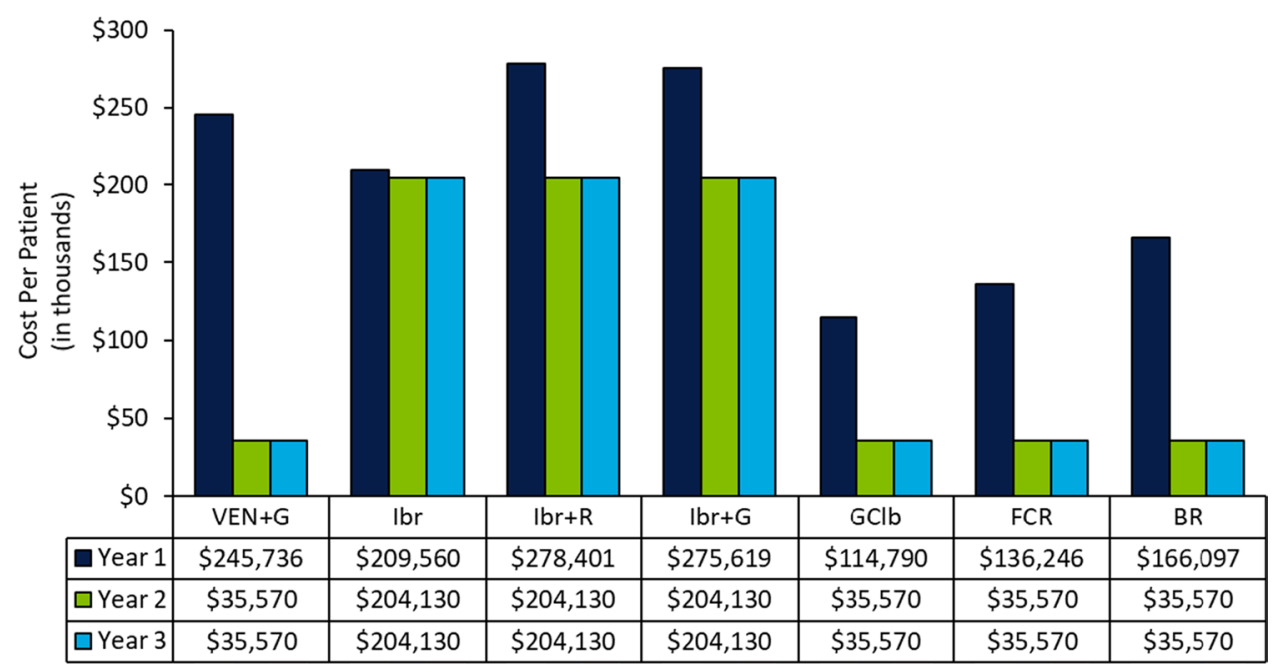


Fig. 3 Comparison of cumulative total cost of care per patient per regimen over 3 years. $B R$ bendamustine + rituximab, $F C R$ fludarabine + cyclophosphamide + rituximab, $G$ Gazyva ${ }^{\circledR}$ (obinutuzumab), GClb obinutuzumab + chlorambucil, $\mathrm{Ibr}$ ibrutinib, $R$ rituximab, VEN venetoclax

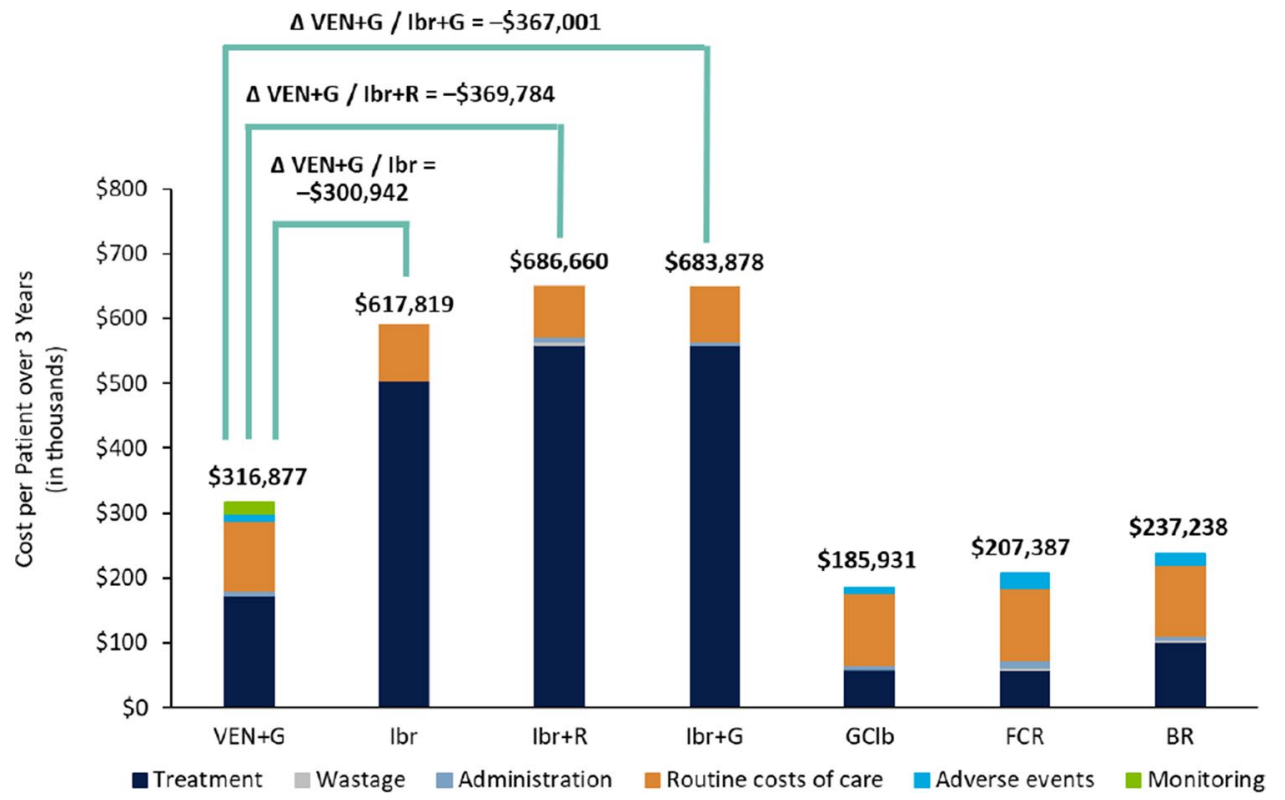

More specifically, extending the time horizon to 5 years increased the cost saving with VEN $+\mathrm{G}$ from $\$ 1,550,663$ to $\$ 12,460,308$, which was a direct result of its 12 -month fixed duration compared to Ibr-based regimens. Another important scenario related to restricting the treatment duration of Ibr to circumvent the lack of progression in the model's base case. Applying conservative assumptions for the duration of Ibr treatment (i.e., 18, 24, and 30 months) still resulted in VEN+G being cost saving in all three Ibr treatment durations tested, with PMPM budget impacts of $-\$ 0.0171,-\$ 0.0380$, and $-\$ 0.0403$, respectively. The scenario where PFS and OS are modeled to define treatment duration and costs reduced the total costs saving associated with VEN+G from $\$ 1,550,663$ to $\$ 1,175,815$
(PMPM budget impact of $-\$ 0.0327$ ). When we penalized VEN $+\mathrm{G}$ costs by assuming all medium TLS risk patients were hospitalized, the PMPM budget impact was reduced from $-\$ 0.0431$ to $-\$ 0.0408$. Also, increasing the proportion of Medicare patients in the health plan to $80 \%$ increased the cost savings to $\$ 4,396,924$ (PMPM budget impact of $-\$ 0.1221)$ as it increased the size of the target population (from 79 to 226 patients in year 1). Similarly, the total costs saving associated with VEN+G varied from $\$ 601,569$ to $\$ 5,345,338$ when restricting the population to Commercial patients only versus Medicare patients only, respectively. As explained, this is due to the size of the target population: from 30 patients (100\% Commercial) to 275 patients $(100 \%$ Medicare) in year 1 versus 79 in the base case $(80 \%$

Table 4 Key scenario analyses

\begin{tabular}{lll}
\hline Scenario & Budget impact & PMPM budget impact \\
\hline Base case & $-\$ 1,550,663$ & $-\$ 0.0431$ \\
$\quad$ Scenario 1: 5-year time horizon & $-\$ 12,460,308$ & $-\$ 0.2077$ \\
Scenario 2: Treatment duration of Ibr-based regimens limited to: & & $-\$ 0.0171$ \\
$\quad 18$ months & $-\$ 615,315$ & $-\$ 0.0380$ \\
24 months & $-\$ 1,368,537$ & $-\$ 0.0403$ \\
30 months & $-\$ 1,452,595$ & $-\$ 0.0327$ \\
Scenario 3: Inclusion of PFS/OS & $-\$ 1,175,815$ & $-\$ 0.0408$ \\
Scenario 4: TLS: $100 \%$ of medium TLS risk patients hospitalized & $-\$ 1,469,321$ & $-\$ 0.1221$ \\
Scenario 5: Payer channel: $80 \%$ Medicare patients/20\% Commercial & $-\$ 4,396,924$ & $-\$ 0.0167$ \\
Scenario 6: Payer channel: $100 \%$ Commercial & $-\$ 601,569$ & $-\$ 0.1485$ \\
Scenario 7: Payer channel: $100 \%$ Medicare & $-\$ 5,345,338$ & $-\$ 0.0238$ \\
Scenario 8: Big 4 prices & $-\$ 858,534$ & \\
\hline
\end{tabular}

Ibr-based regimens: Ibr, Ibr+G, and Ibr+R

$G$ Gazyva ${ }^{\circledR}$ (obinutuzumab), Ibr Ibrutinib, $O S$ overall survival, $P F S$ progression-free survival, $R$ rituximab, $T L S$ tumor lysis syndrome 
Commercial). Finally, in the scenario using Big4 prices that are lower than WAC prices, the PMPM budget impact was reduced to $-\$ 0.0238$.

The one-way sensitivity analyses are depicted in Fig. 4. Across all instances, the inclusion of $\mathrm{VEN}+\mathrm{G}$ as a new treatment option for first-line CLL was associated with cost savings. The PMPM budget impact ranged from $-\$ 0.0631$ and $-\$ 0.0095$, compared to $-\$ 0.0431$ in the base case. The model was most sensitive to parameters related to the costs of venetoclax (treatment and TLS monitoring), and to body surface area, as it impacts the dosing of comparators. More specifically, if all patients had a high tumor burden at baseline, or if TLS monitoring costs increased, the cost savings associated with VEN+G would be reduced, as this would impact the treatment costs of venetoclax. On the other hand, body surface area does not affect the treatment costs of venetoclax but impacts comparators' dosing (i.e., Ibr+R, BR, and FCR). The higher dosing would translate to higher treatment costs, hence increasing $\mathrm{VEN}+\mathrm{G}$ cost savings relative to the base case value.

The scenario analyses and one-way sensitivity analyses further strengthened the base case findings that VEN+G was cost saving.

\section{Discussion}

Budget impact analyses are an essential part of a comprehensive economic assessment of a healthcare intervention and are increasingly required as part of coverage and formulary decisions. We developed a BIM to estimate the cost of introducing $\mathrm{VEN}+\mathrm{G}$ for first-line CLL treatment of patients in a hypothetical US health plan, which included both Medicare and Commercial patients.

The projected treatment landscape included relevant comparators to $\mathrm{VEN}+\mathrm{G}$ in this indication, namely $\mathrm{GClb}$, Ibr, Ibr+R, Ibr+G, FCR, and BR therapy. Cost categories included treatment costs, administration costs, wastage costs, monitoring costs, $\mathrm{AE}$ costs, and routine costs of care. Costs were estimated from various US-based sources. Analyses were performed on a hypothetical health plan of $1,000,000$ members.

In the projected scenario where $\mathrm{VEN}+\mathrm{G}$ was introduced, $\mathrm{VEN}+\mathrm{G}$ use was expected to reach $21.4 \%$ by year 3 . The budget impact analysis estimated a cost saving of $\$ 1,550,663$ over the 3-year time horizon associated with the introduction of VEN+G for first-line CLL treatment, compared to an alternative scenario without $\mathrm{VEN}+\mathrm{G}$. The average PMPM budget impact over this period was $-\$ 0.04$. The lower cumulative treatment cost associated with $\mathrm{VEN}+\mathrm{G}$, which was administered for a fixed 12-month duration, compared to Ibr-based regimens, where Ibr was administered continuously in the base case, was a key driver for this budget reduction. Extensive scenario analyses, including the use of PFS/ OS to define treatment duration and costs, supported the base case finding.

We compared our results to recent US economic studies of CLL treatment costs. Shanafelt et al. [8] estimated that the 10-year pharmaceutical costs would increase by 3.6-fold per treated patient if Ibr replaced FCR/BR as first-line treatment for CLL. In our BIM, over a 5-year horizon, the TCC for Ibr monotherapy was between 3.3 and 3.7 times more than FCR/ BR. Nabhan et al. [29] analyzed a US payer claims registry to compute PMPM healthcare resource utilization of newly

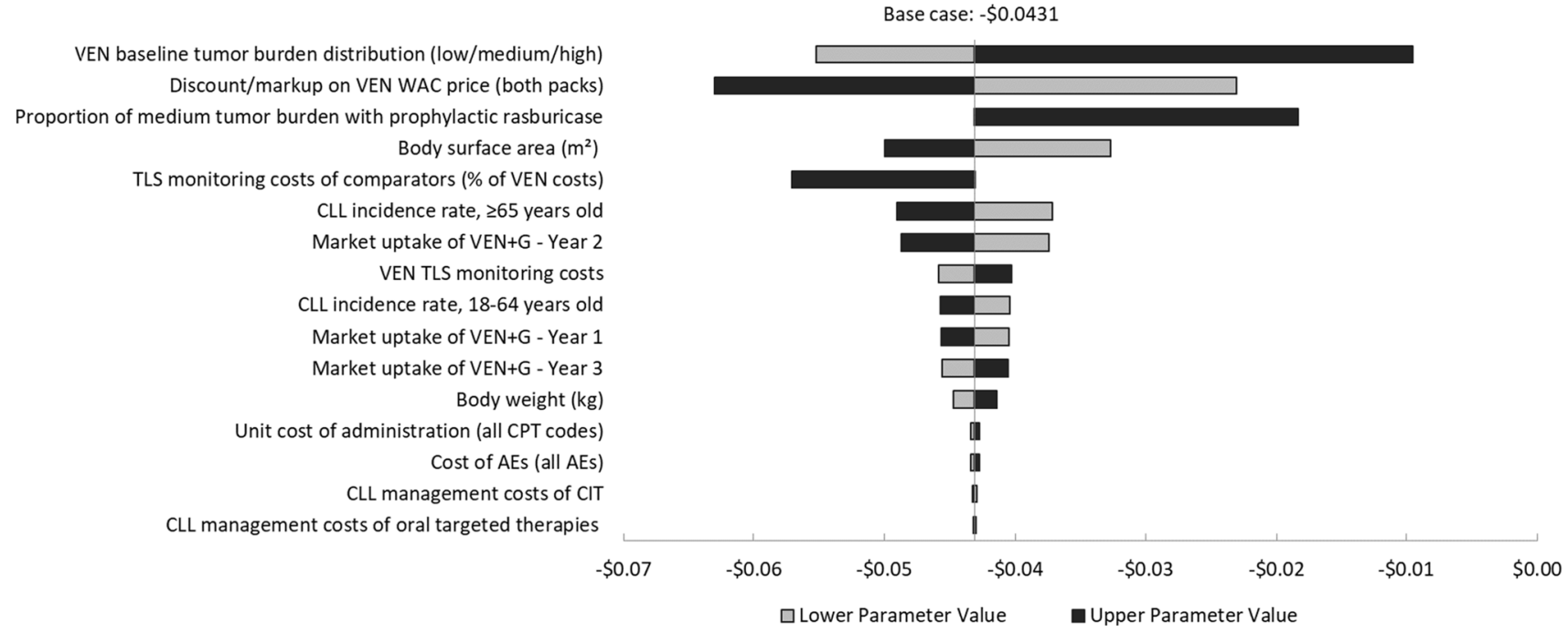

Fig. 4 Tornado diagram of one-way sensitivity analyses on the PMPM budget impact over 3 years. $A E$ adverse event, $C I T$ chemoimmunotherapy, $C L L$ chronic lymphocytic leukemia, $C P T$ Current Pro- cedural Terminology, $G$ Gazyva ${ }^{\circledR}$ (obinutuzumab), $P M P M$ per-member per-month, TLS tumor lysis syndrome, VEN venetoclax, WAC wholesale acquisition cost 
diagnosed CLL patients during their first cycle of therapy. Healthcare resource utilization (number of outpatient and office visits per month) and costs (inpatient hospital stay) were significantly higher in patients treated with chemoimmunotherapy compared to Ibr-treated ones [29]. However, these findings were limited to the first cycle of therapy for CLL, and longer follow-up is needed to inform the true economic impact of each therapy on patient outcomes.

Kabadi et al. [30] analyzed treatment patterns of privately insured, newly diagnosed CLL patients in the US from 2012 to 2015. The authors found that BR was the most common first therapy, while Ibr monotherapy was most commonly used as second and third lines of therapy [30]. Mean monthly per-patient costs (inflated to 2019 USD) for BR, regardless of line of therapy, was $\$ 15,737$ [30]. This was $13 \%$ higher than the (first year) average monthly TCC of $\$ 13,974$ for first-line BR treatment in our model (assuming 100\% Commercial). For Ibr monotherapy, the difference in monthly costs between Kabadi et al. and the current BIM was 34\%, $\$ 23,397$ versus $\$ 17,463$, respectively. The larger difference between the two estimates is likely due to Ibr monotherapy in Kabadi et al. [30] being the second/third therapy (i.e., used in harder to treat patients), whereas our model assumed first-line Ibr monotherapy treatment.

One of the key limitations of our base case analysis was the absence of PFS and OS effects from the patient disease pathway. This was done to retain transparency and simplicity regarding cost elements. The absence of PFS and OS effects meant that for Ibr-based regimens, Ibr was administered continuously throughout the model horizon. That said, in RESONATE-2, the median Ibr treatment duration for first-line CLL was 28.5 months (with a maximum follow-up of 36 months) [15]. This aligns very closely with continuous treatment over the 3-year time horizon. Nonetheless, scenario analyses restricting the duration of Ibr demonstrated that VEN+G remained cost saving, even under a very conservative scenario of 18 -months treatment duration. Furthermore, the inclusion of PFS/OS in a scenario analysis led to similar conclusions where VEN+G remained cost saving.

Second, the budget impact of VEN+G was dependent on its market penetration. These estimates were based on market share projections and hence subject to uncertainty. Sensitivity analyses demonstrated that a greater market penetration for $\mathrm{VEN}+\mathrm{G}$ in years 1 and 2 would further increase cost savings, as the total share of all comparators, including Ibr-based regimens, fell. Another limitation of the model was the assumption of no discontinuation. Consequently, many patients in the model continued treatment for long periods of time, while in real life, discontinuation may occur due to reasons other than progression.

Our model assumed a full vial was used. If the remaining dose were recycled in real-world clinical practice, our model would overestimate the cost of wastage. However, wastage corresponds to a small fraction of TCC, and the impact of this potential overestimation is small. Our base case model assumed the cost of TLS monitoring was only applicable to VEN+G. In scenario analysis, we extended TLS monitoring to other regimens, which further increased the budget saving associated with VEN+G.

Another limitation concerned the source of AE data. Ideally, AE rates would come from head-to-head trials or an indirect treatment comparison to control for differences in trial populations. However, except for $\mathrm{VEN}+\mathrm{G}$ and $\mathrm{GClb}$, $\mathrm{AE}$ rates were taken from different trials. The costs of AEs are derived from HCUP, which reported hospital charges, which were converted to actual hospital expenses to provide care based on the cost-to-charge ratio. The costs may be different from actual reimbursed amounts paid by payers to hospitals. Also, our model assumed the cost of managing AEs was applied once in cycle 1; in real-world practice, patients may experience persistent AEs for the duration of therapy. For these reasons, the cost of managing AEs may be potentially underestimated, especially in Ibr-based regimens. Nonetheless, a sensitivity analysis varying the cost of AEs showed a negligible impact on net budget. Additionally, one of the largest components of TCC was routine costs of care. It is possible that the source for routine cost of care may already have included costs of managing AEs or TLS monitoring. However, the size of bias from this potential overestimation is expected to be small because the TCC for each regimen was primarily driven by drug costs.

Finally, CLL is a disease with remissions, deaths, and recurrences, and a disease state simulation model may be explored in future research to broaden our understanding of the budget implications of various treatment options in CLL.

\section{Conclusions}

The budget impact analysis estimated that the introduction of VEN+G for first-line CLL treatment resulted in substantial cost savings of $\$ 1,550,663$ over the 3-year time horizon for a 1-million-member plan, which translated to an average PMPM budget impact of $-\$ 0.04$ over the same period. The key driver of this cost saving was the fixed 12-month duration of VEN+G compared to Ibr-based regimens, with reductions in cumulative TCC per patient ranging from 49 to $54 \%$. To date, fixed duration treatments were limited to chemoimmunotherapies due to toxicities. Novel agents have better efficacy and safety profiles, but are indicated for treatment until disease progression. $\mathrm{VEN}+\mathrm{G}$ is the first novel agent, only combination regimen, with a fixed duration, which thus fills an important unmet need in CLL treatment, by bringing economic savings for US payers in the era of novel targeted treatments. 
Data Availability Statement The data that support the findings of this study are available in this article. The model is not publicly available due to its intrinsic commercial value and cannot be shared for legal reasons.

\section{Compliance with Ethical Standards}

Disclosures Study conduct, design and financial support were provided by AbbVie Inc. and Genentech. Genentech is a member of the Roche group. AbbVie Inc., Genentech, and Medicus Economics, LLC participated in the design of the research, the analysis and interpretation of findings, and the manuscript writing, review, and approval. All authors contributed to the development of the manuscript and maintained control over the final content.

Conflict of interest Beenish Manzoor, Kavita Sail, Simon Sharmokh, and Tatyana Kapustyan are employees of AbbVie Inc. and may own stocks and/or options of the company. Sang Kyu Cho is a Health Economics and Outcomes Research fellow of AbbVie Inc. Scott Johnson, Suchin Virabhak and Hélène Parisé are employees of Medicus Economics, LLC. Medicus Economics, LLC received consulting fees for research from AbbVie. Arliene Ravelo and Sheila Shapouri are employees of Genentech, Inc. and may own stocks and/or options from Roche. Matthew Davids is an employee of the Dana Farber Cancer Institute. He has appointments for consultancy or is an advisory board member for Genentech, Gilead, Janssen, Astra-Zeneca, Verastem, MEI Pharma, AbbVie, TG Therapeutics, Pharmacyclics, Adaptive Biotechnologies, Sunesis, and Syros; and has institutional research funding from Pharmacyclics, TG Therapeutics, Genentech, Astra-Zeneca, Verastem, BMS, MEI Pharma, and Surface Oncology.

Open Access This article is licensed under a Creative Commons Attribution-NonCommercial 4.0 International License, which permits any non-commercial use, sharing, adaptation, distribution and reproduction in any medium or format, as long as you give appropriate credit to the original author(s) and the source, provide a link to the Creative Commons licence, and indicate if changes were made. The images or other third party material in this article are included in the article's Creative Commons licence, unless indicated otherwise in a credit line to the material. If material is not included in the article's Creative Commons licence and your intended use is not permitted by statutory regulation or exceeds the permitted use, you will need to obtain permission directly from the copyright holder.To view a copy of this licence, visit http://creativecommons.org/licenses/by-nc/4.0/.

\section{References}

1. American Society of Hematology. Leukemia. 2020. https://www. hematology.org/Patients/Cancers/Leukemia.aspx. Accessed 14 Feb 2020.

2. SEER. Cancer Stat Facts: Leukemia-chronic lymphocytic leukemia (CLL). 2019. https://seer.cancer.gov/statfacts/html/clyl.html. Accessed 14 Feb 2020.

3. Eichhorst B, Robak T, Montserrat E, Ghia P, Hillmen P, Hallek $\mathrm{M}$, et al. Chronic lymphocytic leukaemia: ESMO clinical practice guidelines for diagnosis, treatment and follow-up. Ann Oncol. 2015;26(Suppl 5):v78-84. https://doi.org/10.1093/annonc/mdv30 3.

4. Hallek M, Cheson BD, Catovsky D, Caligaris-Cappio F, Dighiero $\mathrm{G}$, Dohner H, et al. iwCLL guidelines for diagnosis, indications for treatment, response assessment, and supportive management of CLL. Blood. 2018;131(25):2745-60. https://doi.org/10.1182/ blood-2017-09-806398.
5. Eichhorst B, Fink AM, Bahlo J, Busch R, Kovacs G, Maurer C, et al. First-line chemoimmunotherapy with bendamustine and rituximab versus fludarabine, cyclophosphamide, and rituximab in patients with advanced chronic lymphocytic leukaemia (CLL10): an international, open-label, randomised, phase 3, non-inferiority trial. Lancet Oncol. 2016;17(7):928-42. https://doi.org/10.1016/ s1470-2045(16)30051-1.

6. Fischer K, Al-Sawaf O, Fink AM, Dixon M, Bahlo J, Warburton $\mathrm{S}$, et al. Venetoclax and obinutuzumab in chronic lymphocytic leukemia. Blood. 2017;129(19):2702-5. https://doi.org/10.1182/ blood-2017-01-761973.

7. Frey S, Blankart CR, Stargardt T. Economic burden and qualityof-life effects of chronic lymphocytic leukemia: a systematic review of the literature. PharmacoEconomics. 2016;34(5):479-98. https://doi.org/10.1007/s40273-015-0367-7.

8. Shanafelt TD, Borah BJ, Finnes HD, Chaffee KG, Ding W, Leis $\mathrm{JF}$, et al. Impact of ibrutinib and idelalisib on the pharmaceutical cost of treating chronic lymphocytic leukemia at the individual and societal levels. J Oncol Pract. 2015;11(3):252-8. https://doi. org/10.1200/jop.2014.002469.

9. Chen Q, Jain N, Ayer T, Wierda WG, Flowers CR, O’Brien SM, et al. Economic burden of chronic lymphocytic leukemia in the era of oral targeted therapies in the United States. J Clin Oncol. 2017;35(2):166-74. https://doi.org/10.1200/JCO.2016.68.2856.

10. National Comprehensive Cancer Network. 2019. NCCN clinical guidelines in oncology: chronic lymphocytic leukemia/small lymphocytic lymphoma. 2.2019 ed2018.

11. Woyach JA, Ruppert AS, Heerema NA, Zhao W, Booth AM, Ding $\mathrm{W}$, et al. Ibrutinib regimens versus chemoimmunotherapy in older patients with untreated CLL. N Engl J Med. 2018;379(26):251728. https://doi.org/10.1056/NEJMoa1812836.

12. Fischer K, Al-Sawaf O, Bahlo J, Fink AM, Tandon M, Dixon M, et al. Venetoclax and obinutuzumab in patients with CLL and coexisting conditions. N Engl J Med. 2019;380(23):2225-36. https://doi.org/10.1056/NEJMoa1815281.

13. Moreno C, Greil R, Demirkan F, Tedeschi A, Anz B, Larratt L, et al. Ibrutinib plus obinutuzumab versus chlorambucil plus obinutuzumab in first-line treatment of chronic lymphocytic leukaemia (iLLUMINATE): a multicentre, randomised, open-label, phase 3 trial. Lancet Oncol. 2019;20(1):43-56. https://doi.org/10.1016/ s1470-2045(18)30788-5.

14. Pharmacyclics LLC. IMBRUVICA®: highlights of prescribing information. 2019.

15. Barr PM, Robak T, Owen C, Tedeschi A, Bairey O, Bartlett NL, et al. Sustained efficacy and detailed clinical follow-up of firstline ibrutinib treatment in older patients with chronic lymphocytic leukemia: extended phase 3 results from RESONATE-2. Haematologica. 2018;103(9):1502-10. https://doi.org/10.3324/haema tol.2018.192328.

16. AbbVie Inc. VENCLEXTA®: highlights of prescribing information. 2019.

17. United States Bureau of Labor Statistics. Consumer price index for all urban consumers: medical care (CUSR0000SAM). 2019. https://www.bls.gov/data/. Accessed 10 Feb 2020.

18. Sullivan SD, Mauskopf JA, Augustovski F, Jaime Caro J, Lee $\mathrm{KM}$, Minchin M, et al. Budget impact analysis-principles of good practice: report of the ISPOR 2012 Budget Impact Analysis Good Practice II Task Force. Value Health. 2014;17(1):5-14. https://doi. org/10.1016/j.jval.2013.08.2291.

19. Howlader N, Noone A, Krapcho M, Miller D, Brest A, Yu M et al. SEER Cancer Statistics Review, 1975-2016, National Cancer Institute. Bethesda, MD, https://seer.cancer.gov/csr/1975_2016/, based on November 2018 SEER data submission, posted to the SEER web site. 2019.

20. US Census Bureau. National Population Projections Datasets, Table 1. Projected Population by Single Year of Age, Sex, Race, 
and Hispanic Origin for the United States: 2016 to 2060. 2017. https://www.census.gov/data/datasets/2017/demo/popproj/2017popproj.html. Accessed 10 Feb 2020.

21. Goede V, Fischer K, Busch R, Engelke A, Eichhorst B, Wendtner $\mathrm{CM}$, et al. Obinutuzumab plus chlorambucil in patients with CLL and coexisting conditions. N Engl J Med. 2014;370(12):1101-10. https://doi.org/10.1056/NEJMoa1313984.

22. Genentech Inc. GAZYVA®: highlights of prescribing information. 2017.

23. Teva Pharmaceuticals USA Inc. BENDEKA®: highlights of prescribing information. 2018.

24. Genentech Inc. RITUXAN@: highlights of prescribing information. 2019.

25. Cancer Therapy Evaluation Program (CTEP). Common Terminology Criteria for Adverse Events (CTCAE) v5.0. 2017. https ://ctep.cancer.gov/protocolDevelopment/electronic_applications/ docs/CTCAE_v5_Quick_Reference_8.5x11.pdf. Accessed 18 Feb 2020.

26. Wang S, Lafeuille MH, Lefebvre P, Romdhani H, Emond B, Senbetta $\mathrm{M}$. Economic burden of treatment failure in chronic lymphocytic leukemia patients. Curr Med Res Opin. 2018;34(6):1135-42. https://doi.org/10.1080/03007995.2018.1464904.

27. Sudhapalli P, Piena M, Palaka A, et al. Systematic literature review and network meta-analysis comparing therapies for treatment-naïve patients with Chronic Lymphocytic Leukemia. E-Poster presented at: European Hematology Association Annual Congress; June 2020, Frankfurt, Germany.

28. US Department of Veterans Affairs. National Acquisition Center (CCST): Pharmaceutical Catalog Search. 2020. https://www. vendorportal.ecms.va.gov/nac/Pharma/List. Accessed 30 Jan 2020.

29. Nabhan CCJ, Mato AR, et al. Comparison of costs and health care resource utilization (HRU) in chronic lymphocytic leukemia (CLL) patients treated with front-line ibrutinib or chemoimmunotherapy. Blood. 2017;130:2111.

30. Kabadi SM, Goyal RK, Nagar SP, Kaye JA, Davis KL. Treatment patterns, adverse events, and economic burden in a privately insured population of patients with chronic lymphocytic leukemia in the United States. Cancer Med. 2019. https://doi.org/10.1002/ cam4.2268.

31. Kaiser Family Foundation. Health Insurance Coverage of the Total Population. 2018. https://www.kff.org/other/state-indicator/total -population/?currentTimeframe $=0 \&$ sortModel $=\% 7 \mathrm{~B} \% 22 \mathrm{col}$ Id\%22:\%22Location\%22,\%22sort\%22:\%22asc\%22\%7D. Accessed 11 Feb 2020. 